\title{
On Uncalibrated Motion-Based Inspection for Conveyor-Belt Systems
}

\author{
Adriano Pascoletti \\ Dipartimento di Matematica e Informatica \\ Università di Udine \\ Udine, Italy \\ pascolet@dimi.uniud.it \\ Emanuele Trucco \\ Department of Computing and Electrical Engineering \\ Heriot-Watt University \\ Edinburgh, UK \\ mtc@cee.hw.ac.uk
}

\begin{abstract}
This paper presents an analytical study and several practical results for computing stable reconstructions with uncalibrated, motion-based inspection and conveyor-belt installations. We achieve metric reconstruction with a simple, efficient algorithm and two nonlinear constraints expressing knowledge readily accessible in a real setup. We analyse the stability and accuracy of reconstruction with respect to the system's mathematical structure, pixelisation, image noise, and constraint values. Extensive experiments with simulated and real data have confirmed our analysis in full, and one example is illustrated here.
\end{abstract}

\section{Introduction and related work}

Motion-based reconstruction is a much-studied problem in computer vision (see [1, 8] for extensive reviews) which has not yet come into its own in inspection applications. However, motion-based algorithms capable of reasonably accurate 3-D measurements, requiring no calibration, and using a single off-the-shelf camera would certainly have applicative interest. This paper refers to a realistic inspection setup particularly important for manufacturing and production, i.e. objects moving on a conveyor belt, and study analytically the configurations in which uncalibrated motion analysis could provide reliable, useful reconstructions.

Uncalibrated vision $[4,7]$ has been attracting increasing attention, as calibration can be time-consuming, and forces to take systems off line for possibly long periods. The system we consider exploits the objects' motion as observed by a static, uncalibrated TV camera, using only image correspondences and no knowledge of the sensor. We achieve metric reconstruction through two nonlinear constraints 
expressing information readily accessible in applications. The geometry considered allows us to develop a simple Cartesian framework to analyse reconstruction stability with respect to the system's mathematical structure, pixelisation, image noise, and constraint values.

Not many papers on motion-based reconstruction derive analytic conditions for optimal sensing configurations [8,2], and we are not aware of any detailed study of the specific problem discussed here. Several authors looked into the stability and degeneracy of the fundamental matrix (eg. [6,9]), but not into practical constraints providing metric reconstruction. Other authors studied the effects of quantisation on vision measurements [5]. Danillidis and Nagel [3] examined the relation between error sensitivity and the error norm used in two-view motion estimation, for translational motion and assuming a calibrated camera. Therefore this paper contributes original, practical results, derived analytically and supported by experiments, on the best sensing configurations for uncalibrated, motion-based reconstruction with conveyor-belt systems.

\section{Assumptions and notation}

We assume a pinhole camera with unknown focal $f$, and specify every quantity in a camera reference frame $(O X Y Z)$ such that the image plane has equation $Z=f$.

Conveyor-belt motion. The scene translates rigidly along an unknown direction in space.

Two-view correspondence. Two views of the scene are acquired by a static camera at instants $t_{1}$ and $t_{2}$. A set of interesting points is tracked across the two frames.

Inter-frame constraint. The norm $a$ of the unknown translation vector from time $t_{1}$ to $t_{2} \mathbf{a}=\left(a_{x}, a_{y}, a_{z}\right)$ is known $(\|\mathbf{a}\|=a>0)$. This information is obviously available in a real installation, as the motion of the conveyor belt can be monitored.

Intra-frame constraint. The 3-D distance between two special points is known, but not their absolute nor relative position. Such points are assumed visible in all images, but could change from pair to pair. In a real setup, this is easily achieved by fixing clearly identifiable patterns on the conveyor belt. Let $d$ be the known norm of the unknown distance vector $\mathbf{d}=\left(d_{x}, d_{y}, d_{z}\right)$ between the two special points $(\|\mathbf{d}\|=d>0)$.

Let the two special points at time $t_{1}$ be $\mathbf{w}_{11}$ and $\mathbf{w}_{21}$, and $\mathbf{w}_{12}$ and $\mathbf{w}_{22}$, respectively, at time $t_{2}$. From now on we shall deal with four 3 -D points $\mathbf{w}_{i j}=$ $\left(X_{i j}, Y_{i j}, Z_{i j}\right)(1 \leq i, j \leq 2)$, with the first and second subscript identifying point and frame respectively. Without loss of generality, we let

$$
\mathbf{a}=\mathbf{w}_{i 2}-\mathbf{w}_{i 1} \quad(1 \leq i \leq 2) \quad \mathbf{d}=\mathbf{w}_{2 j}-\mathbf{w}_{1 j} \quad(1 \leq j \leq 2) .
$$

The 3-D points $\mathbf{w}_{i j}$ determine the image points $\mathbf{p}_{i j}=\left(x_{i j}, y_{i j}\right)$, which give rise to four important 2-D quantities

$$
\mathbf{a}^{\prime}=\mathbf{p}_{12}-\mathbf{p}_{11} \quad \mathbf{a}^{\prime \prime}=\mathbf{p}_{22}-\mathbf{p}_{21} \quad \mathbf{d}^{\prime}=\mathbf{p}_{21}-\mathbf{p}_{11} \quad \mathbf{d}^{\prime \prime}=\mathbf{p}_{22}-\mathbf{p}_{12} .
$$


With these assumptions we have

$$
\mathbf{p}_{i j}=\hat{\mathbf{w}}_{i j} f / Z_{i j} \doteq\left(X_{i j}, Y_{i j}\right) f / Z_{i j} .^{1}
$$

\section{Uncalibrated metric reconstruction}

\subsection{Linear and nonlinear constraints}

We can use equation (3), the rigid motion constraint $\mathbf{w}_{11}-\mathbf{w}_{12}-\mathbf{w}_{21}+\mathbf{w}_{22}=0$, our constraints, and Eqs (1) to get a system of five equations (three linear and two nonlinear) involving four unknown ratios $\tilde{z}_{i j} \doteq Z_{i j} / f(1 \leq i, j \leq 2)$ and the focal length $f$. Let $\tilde{\mathbf{z}}$ denote the column vector $\operatorname{col}\left(\tilde{z}_{11}, \tilde{z}_{12}, \tilde{z}_{21}, \tilde{z}_{22}\right)$, and

$$
\mathbf{M}=\left[\begin{array}{cccc}
x_{11} & -x_{12} & -x_{21} & x_{22} \\
y_{11} & -y_{12} & -y_{21} & y_{22} \\
1 & -1 & -1 & 1
\end{array}\right]
$$

We then get

$$
\begin{aligned}
0 & =\mathbf{M} \tilde{\mathbf{z}} \\
a^{2} & =\left\|\mathbf{p}_{i 2} \tilde{z}_{i 2}-\mathbf{p}_{i 1} \tilde{z}_{i 1}\right\|^{2}+f^{2}\left(\tilde{z}_{i 2}-\tilde{z}_{i 1}\right)^{2} \\
d^{2} & =\left\|\mathbf{p}_{2 j} \tilde{z}_{2 j}-\mathbf{p}_{1 j} \tilde{z}_{1 j}\right\|^{2}+f^{2}\left(\tilde{z}_{2 j}-\tilde{z}_{1 j}\right)^{2} .
\end{aligned}
$$

Notice that any choice of $i, j$ leads to the same equations. An obvious necessary condition for the independence of these equations is that $\mathbf{M}$ has full rank (three). In this case, all solutions of equations (5-7) are multiples of a nontrivial solution $\mathbf{b}=\operatorname{col}\left(b_{11}, b_{12}, b_{21}, b_{22}\right)$, and $\tilde{\mathbf{z}}=\mathbf{b} \varphi$ for some scalar $\varphi$. The condition $Z_{i j}>f>0$ implies the existence of a vector $\mathbf{b}$ with strictly positive components and $\varphi>0$ will follow. The knowledge of $\mathbf{b}$ sheds some light on the scene since the $b_{i j}$ 's are the $Z_{i j}$ 's up to the unknown scale factor $f \varphi$. Substituting $\tilde{\mathbf{z}}=\mathbf{b} \varphi$ into equations (6-7) we get two linear equations in the unknowns $\varphi^{2}$ and $(f \varphi)^{2}$ :

$$
\mathbf{Q} \operatorname{col}\left(\varphi^{2},(f \varphi)^{2}\right)=\operatorname{col}\left(a^{2}, d^{2}\right), \quad \mathbf{Q}=\left[\begin{array}{cc}
\left\|\mathbf{p}_{i 2} b_{i 2}-\mathbf{p}_{i 1} b_{i 1}\right\|^{2} & \left(b_{i 2}-b_{i 1}\right)^{2} \\
\left\|\mathbf{p}_{2 j} b_{2 j}-\mathbf{p}_{1 j} b_{1 j}\right\|^{2} & \left(b_{2 j}-b_{1 j}\right)^{2}
\end{array}\right]
$$

Therefore we have

Proposition 1 The 3-D points $\mathbf{w}_{i j}$ can be uniquely reconstructed from the knowledge of their images $\mathbf{p}_{i j}$ and the norms a,d iff $\mathbf{M}$ has full rank and $\mathbf{Q}$ is non singular.

These conditions have an immediate geometric interpretation.

Proposition $\mathbf{2}$ The matrix $\mathbf{M}$ has full rank iff the images $\mathbf{p}_{i j}$ are not collinear and this is in turn equivalent to the condition that the plane determined by the 3-D points doesn't contain the origin.

\footnotetext{
${ }^{1}$ We shall frequently have to consider the orthogonal projection of a 3-D point onto the XYplane. The projection of a generic point $\mathbf{w}$ will be denoted $\hat{\mathbf{w}}$.
} 
Now, it is easily seen that, in the matrix $\mathbf{Q},\|\hat{a}\|^{2}=\left\|\mathbf{p}_{i 2} b_{i 2}-\mathbf{p}_{i 1} b_{i 1}\right\|^{2} \varphi^{2}$ and $a_{z}^{2}=\left(b_{i 2}-b_{i 1}\right)^{2}(f \varphi)^{2}$, and that similar results hold for $\hat{\mathbf{d}}$ and $d_{z}$. Therefore we have the following practical result:

Proposition $\mathbf{3} \mathbf{Q}$ is non singular iff

$$
\frac{\left|a_{z}\right|}{a} \neq \frac{\left|d_{z}\right|}{d} \quad \text { or equivalently } \quad \frac{\|\hat{\mathbf{a}}\|}{a} \neq \frac{\|\hat{\mathbf{d}}\|}{d} .
$$

i.e. iff $\mathbf{a}$ and $\mathbf{d}$ don't form the same angle with the $X Y$ (retinal) plane.

\subsection{A geometric reconstruction algorithm}

Our data are ten real numbers (eight image coordinates and two norms), which, in the generic case (i.e. on an open and dense subset of $\mathbf{R}^{10}$ ), lead to independent constraints $^{2}$. When a solution $\mathbf{b}$ of (5) with positive components ${ }^{3}$ is available, the reconstruction algorithm is trivial: take the positive square roots of the solutions of (8) and reconstruct the $\mathbf{w}_{i j}$ 's as follows:

$$
\mathbf{w}_{i j}=\left[\begin{array}{c}
\mathbf{p}_{i j} b_{i j} \varphi \\
b_{i j} f \varphi
\end{array}\right]
$$

The particular structure of $\mathbf{M}$ suggests a simple algorithm yielding $\mathbf{b}$ as wedge products of pairs of sides of the quadrilateral determined by the images.

Proposition 4 A solution to $\mathbf{M b}=0$ with positive components is

$$
b_{11}=\left|\mathbf{d}^{\prime \prime} \wedge \mathbf{a}^{\prime \prime}\right|, b_{12}=\left|\mathbf{d}^{\prime} \wedge \mathbf{a}^{\prime \prime}\right|, b_{21}=\left|\mathbf{d}^{\prime \prime} \wedge \mathbf{a}^{\prime}\right|, b_{22}=\left|\mathbf{d}^{\prime} \wedge \mathbf{a}^{\prime}\right| .
$$

This algorithm involves very simple operations on image data, and does not require floating point divisions. On the contrary, commonly available numerical routines computing the kernel of a matrix return a normalised vector (unit norm or one unit component), which can lead to large errors when the image points are nearly aligned.

\subsection{Multiple points and long sequences}

In a real situation, many points are likely to require inspection, and long image sequences are available. This redundancy can be exploited by using many points simultaneously to solve an overconstrained version of system (5-7), and by tracking points across many frames to refine the estimates in time $[8,1]$. Notice that two special points suffice to reconstruct metrically any number of tracked points with two frames. Although redundant methods lead to better accuracies than the twoframe, two-point case that we address, our analysis determines best and worst configurations which remain valid for the multiple-point, multiple-frame cases.

\footnotetext{
${ }^{2}$ When the constraints fail to be independent only a partial reconstruction is possible, which can still be useful for shape inspection. A thorough analysis of these cases has been carried out and is not reported here for lack of space.

${ }^{3}$ The assumption $0<f<Z_{i j}$ guarantees that the components of every solution of (5) are non zero and have the same sign.
} 


\section{Stability of reconstruction}

An analysis of the reconstruction errors caused by image perturbations is quite difficult as the solutions of (8) are ratios of differences of quadratic terms which depend on the images both directly and indirectly via b. Stable working conditions, in which the reconstruction parameters $\varphi$ and $f \varphi$ are not too sensitive to image perturbations, are achieved wherever the matrix $\mathbf{Q}$ is far from singular ${ }^{4}$. This section analyses stability with respect to the system's structure, quantisation, image noise, and constraint values.

\subsection{System structure}

Consider the reconstruction parameters $\varphi$ and $f \varphi$ : expressing the elements of $\mathbf{Q}$ as functions of the $3-\mathrm{D}$ data $\mathbf{w}_{11}, \mathbf{a}, \mathbf{d}$, we can write

$\varphi^{2}=\frac{a^{2}\left(\frac{d_{z}}{Z_{11}}\right)^{2}-d^{2}\left(\frac{a_{z}}{Z_{11}}\right)^{2}}{\left\|\frac{f}{Z_{11}} \hat{\mathbf{a}}\right\|^{2}\left(\frac{d_{z}}{Z_{11}}\right)^{2}-\left\|\frac{f}{Z_{11}} \hat{\mathbf{d}}\right\|^{2}\left(\frac{a_{z}}{Z_{11}}\right)^{2}} \quad(f \varphi)^{2}=\frac{d^{2}\left\|\frac{f}{Z_{11}} \hat{\mathbf{a}}\right\|^{2}-a^{2}\left\|\frac{f}{Z_{11}} \hat{\mathbf{d}}\right\|^{2}}{\left\|\frac{f}{Z_{11}} \hat{\mathbf{a}}\right\|^{2}\left(\frac{d_{z}}{Z_{11}}\right)^{2}-\left\|\frac{f}{Z_{11}} \hat{\mathbf{d}}\right\|^{2}\left(\frac{a_{z}}{Z_{11}}\right)^{2}}$

and the focal length

$$
f^{2}=\frac{d^{2}\left\|\frac{f}{Z_{11}} \hat{\mathbf{a}}\right\|^{2}-a^{2}\left\|\frac{f}{Z_{11}} \hat{\mathbf{d}}\right\|^{2}}{a^{2}\left(\frac{d_{z}}{Z_{11}}\right)^{2}-d^{2}\left(\frac{a_{z}}{Z_{11}}\right)^{2}}
$$

Taking into account (9) it is easily seen that all numerators and denominators vanish simultaneously. This fact can be interpreted geometrically: consider a unit sphere centered at $\mathbf{w}_{11}$ and the unit vectors $\mathbf{d} / d$ and $\mathbf{a} / a$. The points $\mathbf{w}_{11} \pm \mathbf{d} / d$ determine two parallels on the sphere and the nonlinear constraints are independent iff a/a doesn't belong to one of those parallels (Figure 1 (a)). This is only

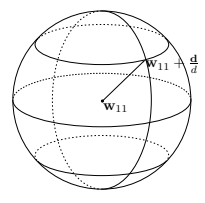

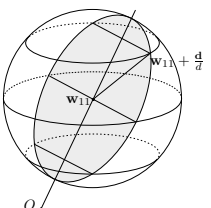

Figure 1: geometric interpretation of reconstruction conditions.

a necessary condition for reconstruction, since the expressions (12-13) are meaningless when $\operatorname{rank}(\mathbf{M})<3$, i.e. when $\mathbf{w}_{11}, \mathbf{w}_{11}+\mathbf{a}, \mathbf{w}_{11}+\mathbf{d}$ determine a plane through the origin or, equivalently, when $\mathbf{w}_{11}+\mathbf{a} / a$ does not lie on the great circle determined on the sphere by $O, \mathbf{w}_{11}$ and $\mathbf{w}_{11}+\mathbf{d} / d$ (Figure $1(\mathrm{~b})$ ). This leads to two results of practical interest:

1. A proximity measure to the condition $\operatorname{rank}(\mathbf{M})<3$ is given by the area of the quadrilateral determined by the images, or, after reconstruction is computed, by the distance from the origin of the plane determined by $\mathbf{w}_{11}$, $\mathbf{w}_{11}+\mathbf{a}, \mathbf{w}_{11}+\mathbf{d}$.

\footnotetext{
${ }^{4}$ Although this is not a complete guarantee of stability, as b is not taken into account.
} 
2. Similarly, a measure of proximity to a situation of dependent nonlinear constraints is given by the absolute value of $\operatorname{det} \mathbf{Q}$ or by ||$a_{z}|/ a-| d_{z}|/ d|$ (see (9)).

\subsection{Quantisation and constraint values}

From a theoretical point of view, reconstruction is possible if and only if $\mathbf{w}_{11}+\mathbf{a} / a$ does not belong either to the parallels determined by $\mathbf{w}_{11} \pm \mathbf{d} / d$ or to the great circle determined by $0, \mathbf{w}_{11}, \mathbf{w}_{11} \pm \mathbf{d} / d$. Extensive simulations have shown that, if no quantisation error (e.g. pixelisation) is introduced, our reconstruction algorithm is quite robust with respect to floating point errors, and accurate reconstruction is achieved even if $\mathbf{a} / a$ is very close to one of the parallels or to the great circle in Figure 1(b).

In real situations, quantisation errors lead to seemingly strange situations. The quadrilateral determined by the images may loose convexity or have a pair of intersecting edges ${ }^{5}$. Another consequence of quantisation errors is the appearance of negative values for $\varphi^{2},(f \varphi)^{2}, f^{2}$. This is due to the fact that the numerators and denominators of (12-13) should be positive whenever $\mathbf{w}_{11}+\mathbf{a} / a$ belongs to the interior of the equatorial strip delimited by the parallels determined by $\mathbf{w}_{11} \pm \mathbf{d} / d$; negative in the gray areas of Figure 2(a). Near the parallels, quantisation errors
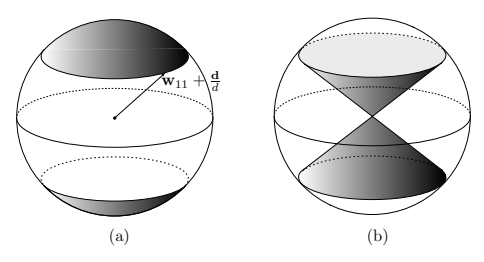

Figure 2: geometric interpretation of quantisation analysis.

can give rise to numerators and denominators of opposite sign. These considerations motivate the search for translations a maximising the absolute values of the numerators and denominators of (12-13), which have the nice property of being monotone functions of $\left|a_{z}\right|$ and reach their maximum all at the same point. We obtain therefore another result of practical interest:

Proposition $\mathbf{5}$ Keeping $\mathbf{d}$, a fixed and varying $\mathbf{a}$, the absolute values of the numerators and denominators of (12-13) are maximized by $a_{z}=0$ if $\left|d_{z}\right| \geq\|\hat{\mathbf{d}}\|$ and by $\left|a_{z}\right|=a$ if $\left|d_{z}\right| \leq\|\hat{\mathbf{d}}\|$.

Similar results hold keeping a fixed and varying d. For instance, Figure 2(b) shows the double cone $\left|Z-Z_{11}\right| \geq \sqrt{\left(X-X_{11}\right)^{2}+\left(Y-Y_{11}\right)^{2}}$. If $\mathbf{w}_{11}+\mathbf{d}$ (resp. $\mathbf{w}_{11}+\mathbf{a}$ ) falls inside it, the best working situation is achieved for $\mathbf{w}_{11}+\mathbf{a} / a$ (resp. $\left.\mathbf{w}_{11}+\mathbf{d} / d\right)$ on the equator and as far as possible from the plane determined by $O, \mathbf{w}_{11}$ and $\mathbf{w}_{11}+\mathbf{d}\left(\right.$ resp. $\left.\mathbf{w}_{11}+\mathbf{a}\right)$. If $\mathbf{w}_{11}+\mathbf{d}\left(\right.$ resp. $\left.\mathbf{w}_{11}+\mathbf{a}\right)$ falls outside the double cone, the best situation is $\mathbf{w}_{11}+\mathbf{a} / a$ (resp. $\mathbf{w}_{11}+\mathbf{d} / d$ ) coinciding with one of the poles, provided that the great circle of Figure 1(b) is not a meridian.

\footnotetext{
${ }^{5}$ Because the components of $\mathbf{b}$ have different signs.
} 
Another interesting question concerns the influence of the norms $a, d$ on the quality of the reconstruction. To tackle this question, Equations (12-13) can be rearranged as the product of two factors: one dependent on $a, d, f, Z_{11}$, the other on the XY- and the Z-components of the unit vectors $\mathbf{a} / a$ and $\mathbf{d} / d$. For instance (13) becomes

$$
f^{2}=\frac{\left(a d \frac{f}{Z_{11}}\right)^{2}}{\left(a d \frac{1}{Z_{11}}\right)^{2}} \cdot \frac{\left\|\frac{\hat{\mathrm{a}}}{a}\right\|^{2}-\left\|\frac{\hat{\mathrm{d}}}{d}\right\|^{2}}{\left|\frac{d_{z}}{d}\right|^{2}-\left|\frac{a_{z}}{a}\right|^{2}} .
$$

Also in the expressions of $\varphi^{2}$ and $(f \varphi)^{2}$ the term $(a d)^{2}$ appears in both numerator and denominator; hence, if $\mathbf{a} / a$ and $\mathbf{d} / d$ are fixed, large values are achieved by making the product ad as large as possible. In this situation the effects of quantisation (pixelisation) errors are minimised. Our simulations showed that reconstruction takes place when ad is bigger than a threshold which is a decreasing function of the pixel sizes. We did not determine a closed-form expression for this threshold, but experimentation confirms that maximising the product ad yields the most stable configurations.

\subsection{Image noise}

It is important to have quantitative estimates of the sensitivity of the reconstructed points to small image perturbations. When the norms $a, d$ and the eight components $x_{i j}, y_{i j}$ are known and the reconstruction is possible, Equation (8) gives $\varphi$ and $f \varphi$, and (10) yields the reconstruction. Let $\xi$ denote one of the quantities $x_{i j}$ or $y_{i j}$. The sensitivities of the reconstructed points to reasonably small perturbations of $\xi$, such as those expected from subpixel-precision feature detectors or trackers, are given by the following formulae derived from (10):

$$
\begin{aligned}
\frac{\partial \hat{\mathbf{w}}_{i j}}{\partial \xi} & =\frac{\partial \mathbf{p}_{i j}}{\partial \xi} b_{i j} \varphi+\mathbf{p}_{i j} \frac{\partial b_{i j}}{\partial \xi} \varphi+\mathbf{p}_{i j} b_{i j} \frac{\partial \varphi}{\partial \xi} \\
\frac{\partial Z_{i j}}{\partial \xi} & =\frac{\partial b_{i j}}{\partial \xi} f \varphi+b_{i j} \frac{\partial f \varphi}{\partial \xi} .
\end{aligned}
$$

The partial derivatives of $\mathbf{p}_{i j}$ are trivial; those of $b_{i j}$ are easily computed from (11); the remaining ones are given by

$$
\operatorname{col}\left(\frac{\partial \varphi}{\partial \xi}, \frac{\partial f \varphi}{\partial \xi}\right)=-\left[\begin{array}{cc}
\frac{1}{2 \varphi} & 0 \\
0 & \frac{1}{2 f \varphi}
\end{array}\right] \mathbf{Q}^{-1} \frac{\partial \mathbf{Q}}{\partial \xi} \operatorname{col}\left(\varphi^{2}, f \varphi^{2}\right)
$$

with $\frac{\partial \mathbf{Q}}{\partial \xi}$ computable from (8).

\section{$5 \quad$ Experimental results}

Extensive experiments have been run on both simulated and real data. Synthetic data simulated a large number of situations by varying the relative position of camera and translation, the constraint values, and the pixelisation level. Here, we discuss only one representative simulation with $\mathbf{w}_{11}=(0,20,200) \mathrm{mm}, a=50 \mathrm{~mm}$, $\mathbf{d}=(0,0,60) \mathrm{mm}$, focal length $50 \mathrm{~mm}$ and pixelisation at $100 \mathrm{pixel} / \mathrm{mm}$. These 
values of $\mathbf{w}_{11}$ and $\mathbf{d}$ allow us to show separately the effects of the proximity of $\mathbf{a} / a$ to the parallels determined by $\pm \mathbf{d} / d$, and to the great circle through $0, \mathbf{w}_{11}$, $\mathbf{w}_{11}+\mathbf{d}$ (Section 4.1). In our case, $\mathbf{w}_{11} \pm \mathbf{d}$ are the poles of the unit sphere centered at $\mathbf{w}_{11}$ and the critical great circle is determined by the $y z$ plane, so that Figure 1 becomes Figure 3(a).
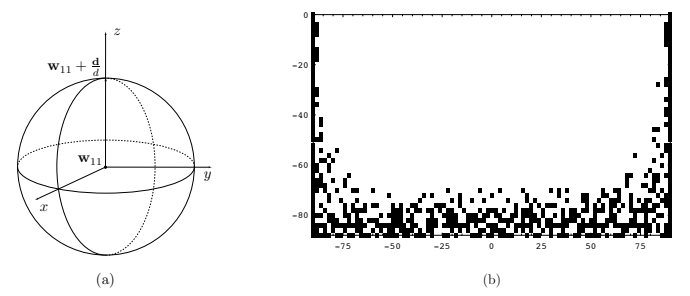

Figure 3: (a) sphere reference frame; (b) sphere points where reconstruction fails.

The proximity of a/a to the poles is measured by $\delta_{p}=|| a_{z}|/ a-| d_{z}|/ d|$ (see (9)) and the proximity to the great circle is measured by $\delta_{0}$, the distance of the plane passing through $\mathbf{w}_{11}, \mathbf{w}_{11}+\mathbf{a}, \mathbf{w}_{11}+\mathbf{d}$ from the origin, or, as well, by the area $\alpha$ of the quadrilateral formed by the four images: $\alpha \doteq \frac{1}{2}\left(\left|\mathbf{a}^{\prime} \wedge \mathbf{d}^{\prime}\right|+\left|\mathbf{a}^{\prime \prime} \wedge \mathbf{d}^{\prime \prime}\right|\right)$. We ran the algorithm for all the nodal points of a grid of meridians and parallels spaced by $2^{\circ}$. Longitudes varied in $\left[-90^{\circ}\right.$ to $\left.+90^{\circ}\right]$, latitudes in $\left[-88^{\circ} \text { to } 0^{\circ}\right]^{6}$. The working points where reconstruction is not possible are shown in Figure $3(\mathrm{~b})$. Three zones are evident: (1) reconstruction fails at longitude $\pm 90^{\circ}$ because $\operatorname{rank}(\mathbf{M})<3$ (Proposition 1), the images are collinear, and both $\alpha$ and $\delta_{0}$ vanish; (2) for latitudes greater than $70^{\circ} \mathrm{S}$, where $\mathbf{a} / a$ is near to the parallel determined by $-\mathbf{d} / d$ (south pole), $\delta_{p}$ is small and pixelisation and floating point errors lead to numerators and denominators of $\varphi^{2},(f \varphi)^{2}, f^{2}$ with different signs; (3) in the remaining zone reconstruction is inhibited by the combined effect of quasi-collinear images and small $\delta_{p}$. If quantisation errors were absent, the reconstruction process would be affected by floating errors only and reconstruction would be possible anywhere with the exception of very small zones around the great circle at longitude $90^{\circ}$ (less than $0.1^{\circ}$ wide) and near the poles (less than $0.5^{\circ}$ ). When reconstruction is possible, the best configurations (yielding best stability and minimum absolute errors) are the ones farthest from the non-reconstruction regions. An interesting quantity is the focal length $f$ : the percentage and the location of points where the relative error $\epsilon_{f}$ on $f$ doesn't exceed a given threshold are summarised in Figure 4 and in the following table:

\begin{tabular}{|lrrrrrrrrrrrr|}
\hline$\left|\epsilon_{f} \%\right| \leq$ & 1 & 2 & 5 & 10 & 15 & 20 & 25 & 30 & 40 & 50 & 75 & 100 \\
\hline$\%$ points & 27 & 40 & 59 & 71 & 78 & 82 & 85 & 86 & 90 & 92 & 95 & 98 \\
\hline
\end{tabular}

It is apparent that small errors are concentrated near the equator in accordance with Proposition 5, the practical importance of which becomes apparent if we inspect the errors obtained for varying latitude and constant longitude. Figure 5

\footnotetext{
${ }^{6}$ Longitudes $90^{\circ}-270^{\circ}$ lead to similar results and nothing worth noticing happens on the northern emisphere w.r.t. the southern one.
} 

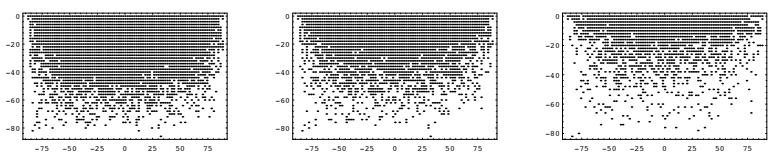

Figure 4: points where $\left|\epsilon_{f}\right| \leq 10 \%, 5 \%, 2 \%$ (left to right).

plots the absolute relative errors on $Y_{12}$ against latitude for three different longitudes $\left(-60^{\circ},-30^{\circ},-10^{\circ}\right)$. The distance $\delta_{p}$ is a convex function of latitude for longitudes between $-90^{\circ}$ and $0^{\circ}$, with a maximum in $0^{\circ}$ and a minimum in $-90^{\circ}$. We limit our attention to the reconstruction errors of $\mathbf{w}_{12}$ because $\mathbf{w}_{11}, \mathbf{w}_{21}$ are less sensitive; $\mathbf{w}_{22}$ behaves much like $\mathbf{w}_{12}$; the $X$-component is affected by smaller errors. Space constraints don't allow us to display results for positive longitudes;
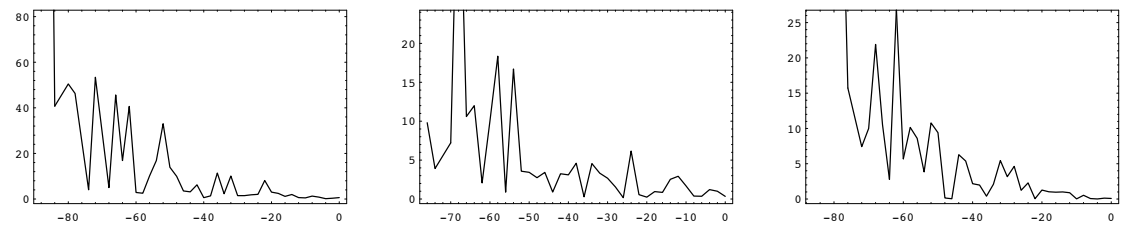

Figure 5: relative errors on $Y_{12}$ at longitudes $-60^{\circ},-30^{\circ},-10^{\circ}$.

in general, an increase in $\delta_{p}$ causes a systematic decrease of the reconstruction errors, and the smallest errors depend on the longitude considered. We observed the effects of the proximity of $\mathbf{a} / a$ to the great circle contained in the $x y$ plane of Figure 3(a) (where the area vanishes) by considering a fixed latitude and varying the longitude from $-90^{\circ}$ to $+90^{\circ}$. Figure 6 shows the strict correlation between the area $\alpha$ and the reconstruction errors on both the spatial components and the focal length; the distance $\delta_{0}$ exhibits a similar behaviour. Our analysis was fully con-
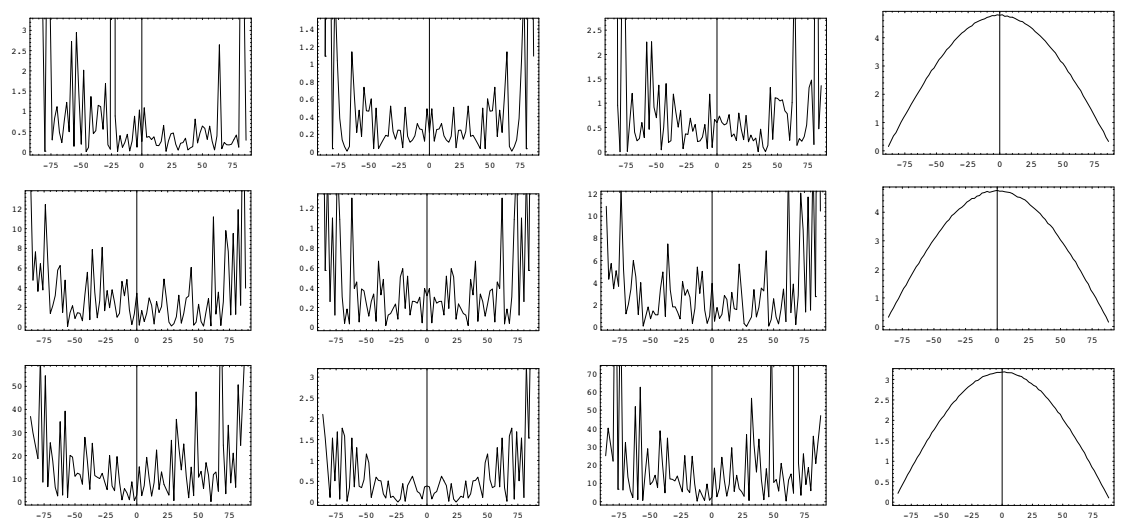

Figure 6: relative errors on $Y_{12}, Z_{12}, f$ and $\alpha$ at latitudes $-10^{\circ}$ (first row), $-30^{\circ},-60^{\circ}$ (last row); longitudes on the horizontal axis.

firmed by real experiments, in which a calibration pattern (two white planes with 
grids of black circles) moved on a computer-controlled translation stage, and we tracked the centers of the circles. Ground truth for error estimation was supplied by our implementation of Tsai's calibration [10].

\section{Discussion}

We have presented a simple algorithm for uncalibrated, motion-based reconstruction with conveyor-belt installations, and derived analytically best-accuracy configurations. We have identified degenerate configurations which could easily occur in a practical setup, and suggested tests for estimating the stability and accuracy of reconstructions. Synthetic and real experiments fully support the theory. Our two-view, two-point stability analysis remains important when multiple correspondences and long sequences are used. Our results are relevant for applications as conveyor-belt systems are very common, and motion-based inspection could be implemented at very low cost.

\section{Acknowledgements}

This work was partially supported by a British Council-MURST/CRUI grant.

\section{References}

[1] J K Aggarwal and N Nandhakumar: On the Computation of Motion from Sequences of Images: a Review, Proc. IEEE 76(8), 1988, pp. 917-935.

[2] S D Blostein and T S Huang: Quantisation Errors in Stereo Triangulation, IEEE Trans Patt Anal Mach Intell 9, 1987, pp. 752-765.

[3] K Danillidis and H-H Nagel: Analytical Results on Error Sensitivity of Motion Estimation from Two Views, Image and Vision Computing 8(4), 1990, pp. 297-303.

[4] O Faugeras: What Can Be Seen in Three Dimensions with an Uncalibrated Stereo Rig?, Proc Eur Conf Comp Vis, S Margherita, 1992, pp. 563-578.

[5] B Kamgar-Parsi and B Kamgar-Parsi: Evaluation of Quantization Errors in Computer Vision, IEEE Trans Patt Anal Mach Intell 11(9), 1989, pp. 929-939.

[6] Q-T Luong and O Faugeras: The Fundamental Matrix: Theory, Algorithms, and Stability Analysis, Int Journ Comp Vis 1(17), 1996, pp. 43-76.

[7] N Hollighurst and R Cipolla: Uncalibrated Stereo Hand-Eye Coordination, Image and Vision Computing 12(3), 1994, pp. 187-192.

[8] T S Huang and A N Netravali: Motion and Structure from Feature Correspondence: a Review, Proc. IEEE 82(2), 1994, pp. 252-267.

[9] P H S Torr, A Zisserman and S J Maybank: Robust Detection of Degenerate Configurations for the Fundamental Matrix, Proc IEEE Int Conf Comp Vis, Cambridge (MA), 1995, pp. 1037-1042.

[10] R Y Tsai: A Versatile Camera Calibration Technique for High Accuracy 3D Machine Vision Metrology, IEEE Journ. Robot. Autom. 3(4), 1987, pp. 323-344. 